\title{
Research on Comprehensive Energy Saving Control Strategy of Air Conditioning
}

\author{
Guozeng $\mathrm{Wu}^{1,2}$, Tao $\mathrm{Li}^{1,2}$, Yijin Gang ${ }^{1,2}$ \\ ${ }^{1}$ School of Human Settlements and Civil Engineering, Xi'an Jiaotong University, Xi'an, Shaanxi, China \\ ${ }^{2}$ SIPPR Engineering Group Co., Ltd, Zhengzhou, Henan, China
}

\begin{abstract}
:
On the basis of ensuring the requirements of process air parameters, the air conditioning control should reduce the energy consumption of the air conditioning system to the maximum extent. In this paper, by improving the adjusting speed and stability of the air conditioning system, and according to the process of environmental indicators allow deviation of belt, on the premise of not beyond the maximum technical index requirements by control algorithm to achieve better energy saving effect.
\end{abstract}

Keywords: BAS, Air conditioning system, PID.

\section{INTRODUCTION}

In today's society where energy demand is increasing and energy is increasingly tight, energy-saving technologies are increasingly being valued by all sectors of society. In the industrial field, production workshops have high requirements for air parameters, and generally require constant temperature and humidity air conditioning, such as cigarette factories, pharmaceutical factories, etc[1]. On the one hand, high-quality air conditioners meet the requirements of the production process, but on the other hand they also cause higher energy consumption.

According to relevant data, compared with civil buildings with the same air-conditioning area, the air exchange frequency of the workshop process air-conditioning system is 2.0-2.5 times that of civil buildings[2-3]. Large-capacity air-conditioning systems lead to high operating costs. According to incomplete statistics, the energy (electricity, boiler fuel) consumed by the 
air-conditioning system every year accounts for about $40 \%$ of the plant's public power consumption[3-4].In the current engineering design, an automatic control system is generally used to manage the process air conditioner. How to minimize the energy consumption of the process air conditioner system on the basis of ensuring the air requirements of the workshop has become the focus of the automatic control system design[4-5].

In this paper, by improving the adjustment speed and stability of the air conditioning system, and according to the allowable deviation band of the process environment index, the control algorithm is used to achieve a better air conditioning energy saving effect without exceeding the maximum process index requirement[5-7].

\section{HIGH PRECISION CONSTANT TEMPERATURE AND HUMIDITY CONTROL STRATEGY}

The primary index of the workshop process air conditioning control system is to continuously and uninterruptedly meet the high-precision constant temperature and humidity requirements in the production process, and can quickly and effectively overcome various external interferences. At the same time, system control accuracy is also one of the important index to measure the energy-saving efficiency of air-conditioning systems. Therefore, according to the actual environment of the industrial workshop and the control accuracy requirements of temperature and humidity, the following ideas are adopted to control the workshop process air conditioning system[8].

\section{A. Variable parameter adaptive PID control strategy}

The principle block diagram of process air conditioning control is shown in Figure 1.

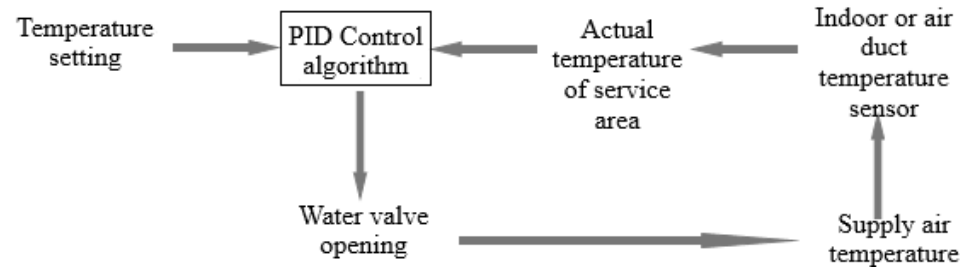

Figure 1:Block diagram of process air conditioning control principle

The core of the high-precision temperature and humidity control strategy of the process air conditioner inherits the classic PID closed-loop control algorithm, the control effect of the traditional PID control algorithm depends on the correct matching of P (proportional) I (integral) 
and D (derivative) parameters, unreasonable parameter settings will cause fluctuations and oscillations in control, and reduce control accuracy and stability. In the actual workshop environment, the working conditions of the air conditioning system are constantly changing throughout the year, due to the dynamic thermophysical properties of the workshop and changing outdoor weather conditions in different seasons, the proportional and integral constants of the temperature and humidity control loop should also be dynamically changed to prevent large overshoot and infinite oscillation. On the other hand, when the air-conditioning system just starts to run, due to the large difference between the target value and the actual value, it is necessary to quickly adjust the PID of the air-conditioning system. After the system is stable, in order to maintain the stability of the system, the air conditioning system requires the PID adjustment of the system not to be too sensitive to prevent unnecessary disturbance[9].

Therefore, this article adopts a variable parameter adaptive PID control strategy, this control strategy adjusts the PID control parameters of the system under different working conditions, so that the air-conditioning system can not only respond quickly, but also ensure high control accuracy.

Using Ziegler-Nichols self-tuning algorithm, its working principle is:

First set the integral and derivative gains to 0 , then the proportional gain gradually increases from zero until it reaches the limit gain $\mathrm{KU}$, at which time the controller output value oscillates at a constant value. According to different types, KU and oscillation period TU automatically calculate proportional, integral and derivative gains under different working conditions according to the methods in TABLE I.

TABLE I. Ziegler Nichols self-tuning algorithm

\begin{tabular}{|c|c|c|c|}
\hline CONTROL TYPE & $\mathrm{Kp}$ & $\mathrm{Ki}$ & $\mathrm{Kd}$ \\
\hline PROPORTIONAL-INTEGRAL & $\mathrm{Ku} / 2.2$ & $1.2 \mathrm{Kp} / \mathrm{Tu}$ & \\
\hline PROPORTIONAL-INTEGRAL-DERIVATIVE & $0.6 \mathrm{Ku}$ & $2 \mathrm{Kp} / \mathrm{Tu}$ & $\mathrm{KpTu} / 8$ \\
\hline
\end{tabular}

The control principle block diagram is shown in Figure 2. 


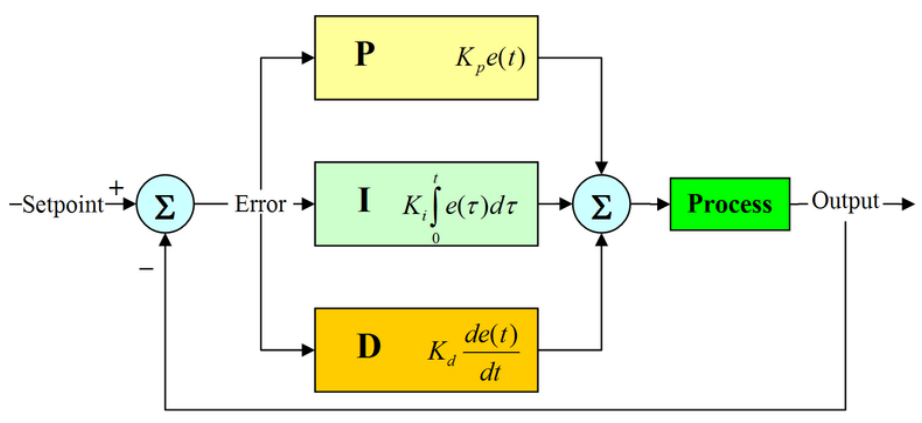

Figure 2:PID control principle block diagram

\section{B. Anti-disturbance control strategy of cascade regulation}

In the environment of industrial workshops, joint workshops, etc. the controlled area of process air-conditioning is often large, and the temperature and humidity of the air, especially the temperature, is a slowly changing process. According to the traditional single-loop classic feedback control theory, adjustment is gradually made only when the controlled object has a deviation, that is, the temperature and humidity deviate from the set value. This feedback adjustment needs to be adjusted after the workshop temperature and humidity sensor data is collected, and there is often a large lag, which leads to fluctuations in temperature and humidity[10].

In order to prevent the temperature and humidity overshoot caused by the lag time, this paper uses the air supply temperature and humidity parameters as intermediate variables for cascade feedback control, The control principle is shown in Figure 3.(Take the relative humidity control loop as an example)

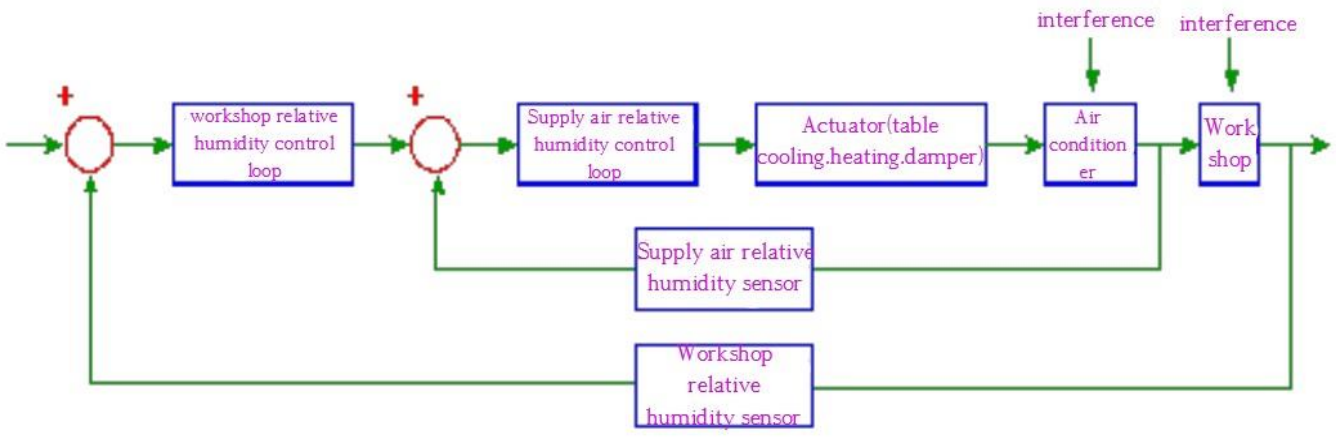


Figure 3:Block diagram of cascade feedback control

As can be seen from Fig.3, When the outdoor environment changes, the heat source or the cold source changes suddenly, the air temperature and humidity will change first, under the feedforward compensation strategy of cascade feedforward control, the control system will automatically compensate and overcome the disturbance of the supply air temperature and humidity in advance, instead of the traditional feedback control theory that requires the temperature and humidity of the air-conditioning area to change before taking control compensation. The cascade regulation will effectively avoid and reduce the influence of interference on the temperature and humidity fluctuations of the controlled air-conditioning area.

\section{Variable integral anti-saturation optimization control strategy}

An important factor that affects the control accuracy and stability of the process air conditioning control system is that the temperature and humidity deviations at the beginning of the air conditioner are large, the temperature and humidity control is inert, and the rise time is slow, and it is easy to cause the integral over-saturation of the control process. Too much integral leads to too much overshoot and the problem of instability for long time. Figure 4(b) shows the integral saturation, (c) is the integral unsaturated condition.
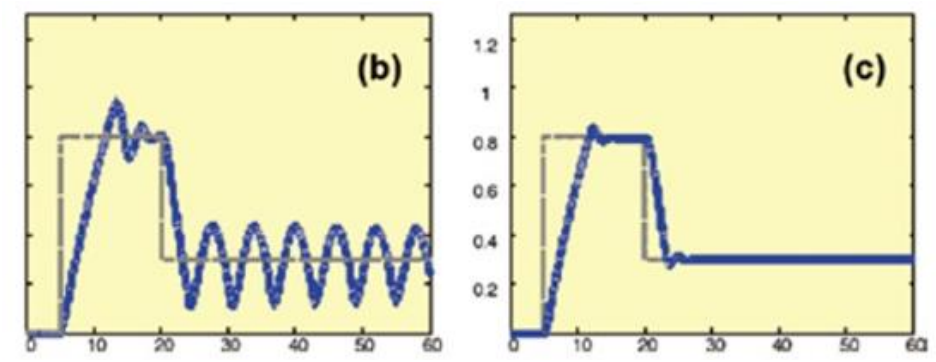

Figure 4:Comparison of PID control with or without oversaturation

In response to this problem, this article uses integral separation methods to prevent the occurrence of integral oversaturation, that is, when the controlled variable starts to track, until the controlled variable is close to the new given value, the integral calculation is started to avoid system oscillation caused by integral oversaturation and overshoot, and improve system response time and stability. 


$$
u(k)=K_{p} e(k)+K_{I} \sum_{j=0} K_{l} e(j)+K_{D}(e(k)-e(k-1))
$$

among them

$$
\mathrm{K}_{1}=\left\{\begin{array}{l}
1 \text { when } \mathrm{e}(\mathrm{k}) \leq \varepsilon \\
0 \text { when } \mathrm{e}(\mathrm{k})>\varepsilon
\end{array}\right.
$$

As can be seen from Figure 5. after using the integral separation algorithm, the system response time and stability are effectively improved.

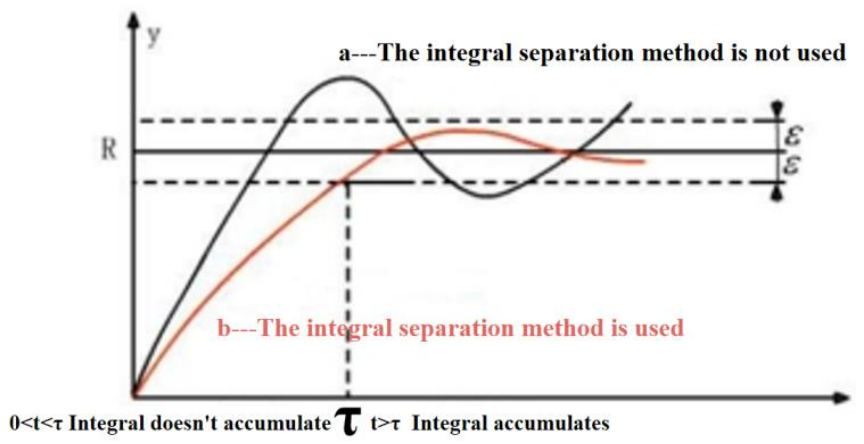

Figure 5:Control effect diagram before and after using integral separation algorithm

In addition, the threshold of points separation should be determined on the spot depending on the specific object and requirements. If the threshold is too high for the purpose of integral separation, if it is too small, it is possible that the controlled quantity cannot jump out of the integral separation zone, resulting in residual errors.

\section{DYNAMIC SETTING VALUE ADAPTIVE ENERGY-SAVING CONTROL STRATEGY}

The traditional constant temperature and humidity air conditioning control algorithm model focuses on how to most accurately control the temperature and humidity of the controlled area at the intermediate value set by the user, for example, it is required to control the temperature in summer at $25 \pm 2{ }^{\circ} \mathrm{C}$ and the humidity at $60 \pm 5 \%$, in winter the temperature at $24 \pm 2{ }^{\circ} \mathrm{C}$ and the humidity at $60 \pm 5 \%$. The ideal result of adopting the traditional air-conditioning control model is to control the temperature and humidity at 25 degrees and $60 \%$ in summer and 24 degrees and $60 \%$ in winter, and strive for accurate constant temperature and humidity for on-site temperature and humidity control. 
The core of the energy-saving control model with automatic variable set point is to make reasonable use of the deviation band allowed by the process environmental indicators, under the premise of not exceeding the requirements of the maximum process index, better air conditioning energy-saving effects can be achieved by controlling as close as possible to the lower limit in winter and as close to the upper limit as possible in summer.

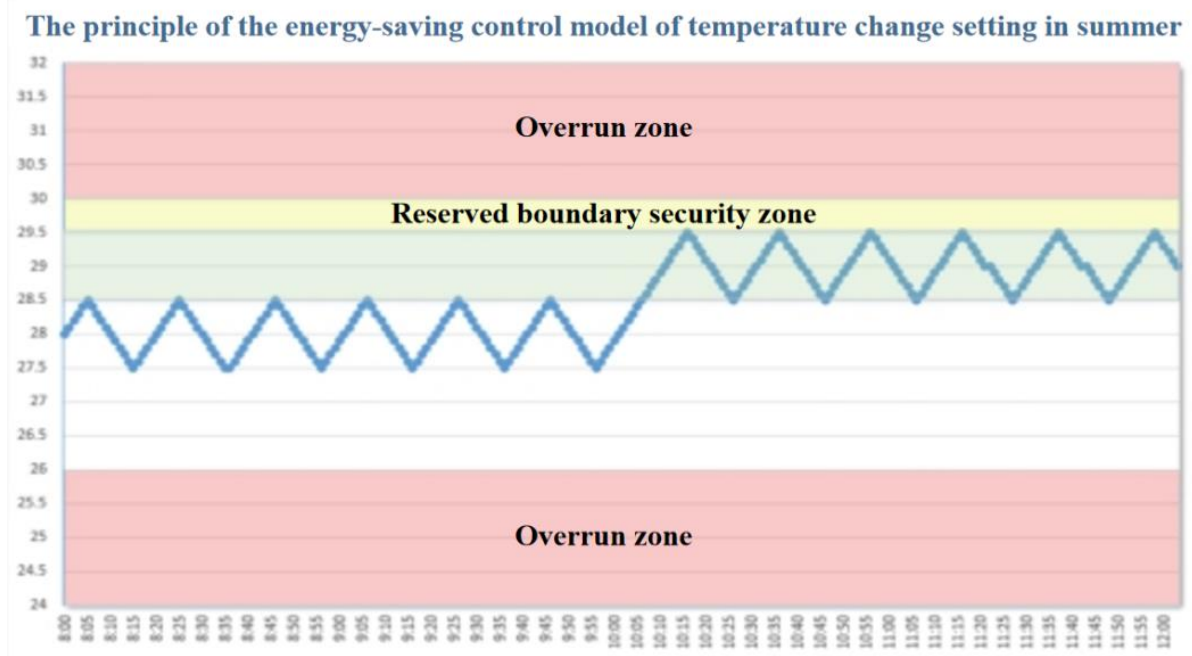

Figure 6:Energy-saving control model of temperature change setting in summer

In the summer cooling mode, it is assumed that the target temperature of the air conditioning control system is 28 degrees, and the maximum fluctuation of the ambient temperature in the past 2 hours is \pm 0.5 degrees. Adopt the energy-saving control model of automatically variable setting value,the automatic control system will automatically gradually increase the temperature setting value to 29 degrees after analyzing the trend changes in the past 2 hours, then began to continuously detect and analyze the new temperature fluctuation range, assuming that the maximum temperature fluctuation observed after a period of time is still \pm 0.5 degrees, the control system will maintain the new temperature setpoint closer to the upper limit of the setpoint; On the contrary, if overshoot occurs, the corrected temperature setting value gradually returns to the temperature center setting value, finally, it is guaranteed that the reserved safety boundary value $>=$ the maximum fluctuation value. Throughout the control process, the temperature setting value of the control system dynamically changes gradually according to the stability of the ambient temperature, in the end, the temperature setting value can be adaptively increased as much as possible while ensuring that sufficient boundary safety is not exceeded, thereby reducing the demand for cooling capacity and achieving the purpose of energy saving for air conditioning. 


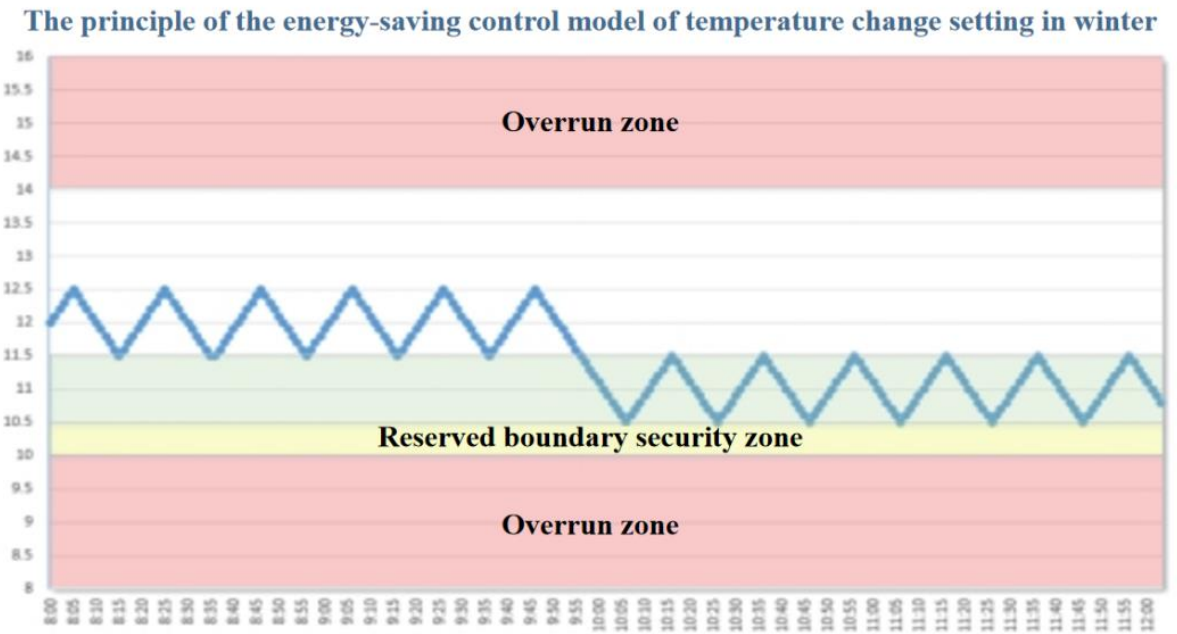

Figure 7:Energy-saving control model of temperature change setting in winter

In the winter heating mode, it is assumed that the temperature target value of the air conditioning control system is 12 degrees, and the maximum fluctuation of the ambient temperature in the past 2 hours is \pm 0.5 degrees. Adopt an innovative energy-saving control model that automatically changes the set value, after the automatic control system analyzes the trend change in the past 2 hours, it will automatically reduce the temperature setting value gradually by 1 degree, and then continue to detect and analyze the new temperature fluctuation range,assuming that the maximum temperature fluctuation observed after a period of time is still \pm 0.5 degrees, the control system will maintain the new temperature set value closer to the lower limit of the set value; On the contrary, if overshoot occurs, the corrected temperature setting value will gradually return to the temperature center setting value, and finally ensure that the reserved safety boundary value $>=$ the maximum fluctuation value.During the whole control process, the temperature setting value of the control system changes slowly and gradually according to the stability of the ambient temperature, in the end, the temperature setting value can be adaptively lowered as much as possible while ensuring that sufficient boundary safety is not exceeded, so as to reduce the demand for heating and achieve the purpose of energy saving for air conditioning. 


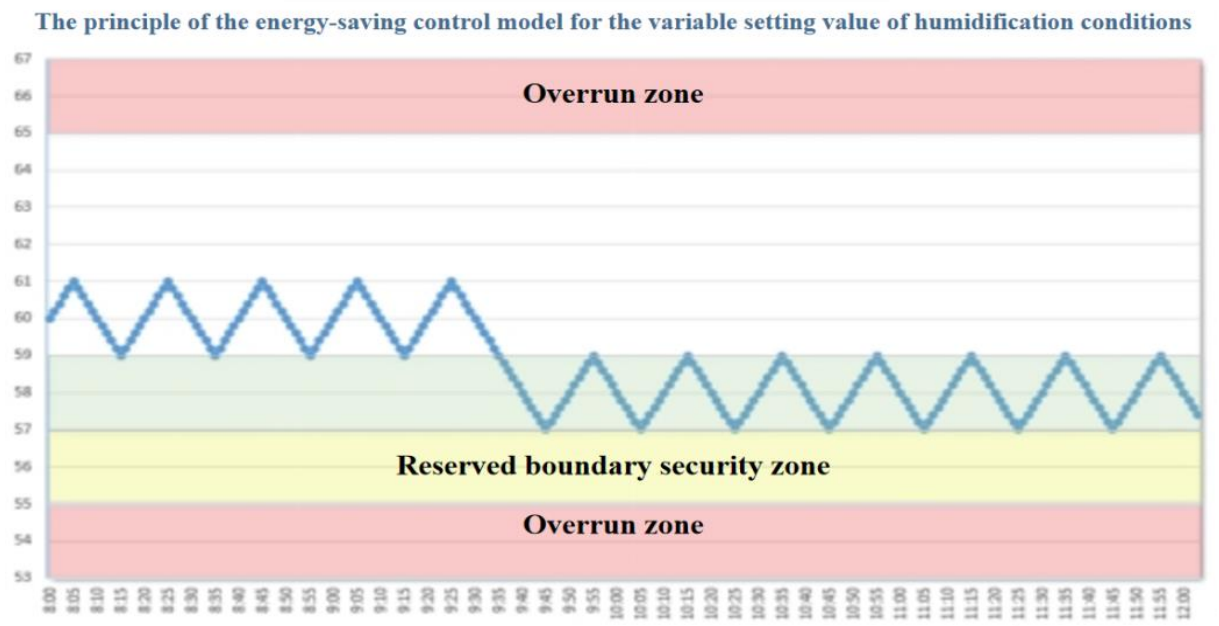

Figure 8:Energy-saving control model for the variable setting value of humidification conditions

In humidification mode, the target central value of humidity control of the air conditioning control system is $60 \%$, assume that the maximum fluctuation range of the system humidity in the past 2 hours is $\pm 1 \%$. Using the automatic variable set value energy-saving control model, the automatic control system will gradually and slowly reduce the humidity set value to $58 \%$ after analyzing the trend change in the past 2 hours, Then began to continuously check and analyze the fluctuation trend of environmental humidity, assuming that the maximum humidity fluctuation observed after a period of time is still $\pm 1 \%$, then the adjusted new setting value closer to the lower limit of the humidity setting value will be maintained; On the contrary, if the humidity is over-adjusted, the humidity setting value will automatically return to the center setting value step by step, and finally ensure that the reserved safety boundary value $>=$ maximum wave range. During the entire control process, the humidity setting value of the control system is dynamically and slowly gradually changed according to the stability of the environmental humidity, in the end, the system humidity setting value can be adaptively decreased as much as possible while ensuring that sufficient boundary safety is not exceeded, so as to reduce the demand for humidification to achieve the purpose of energy saving for air conditioning. 


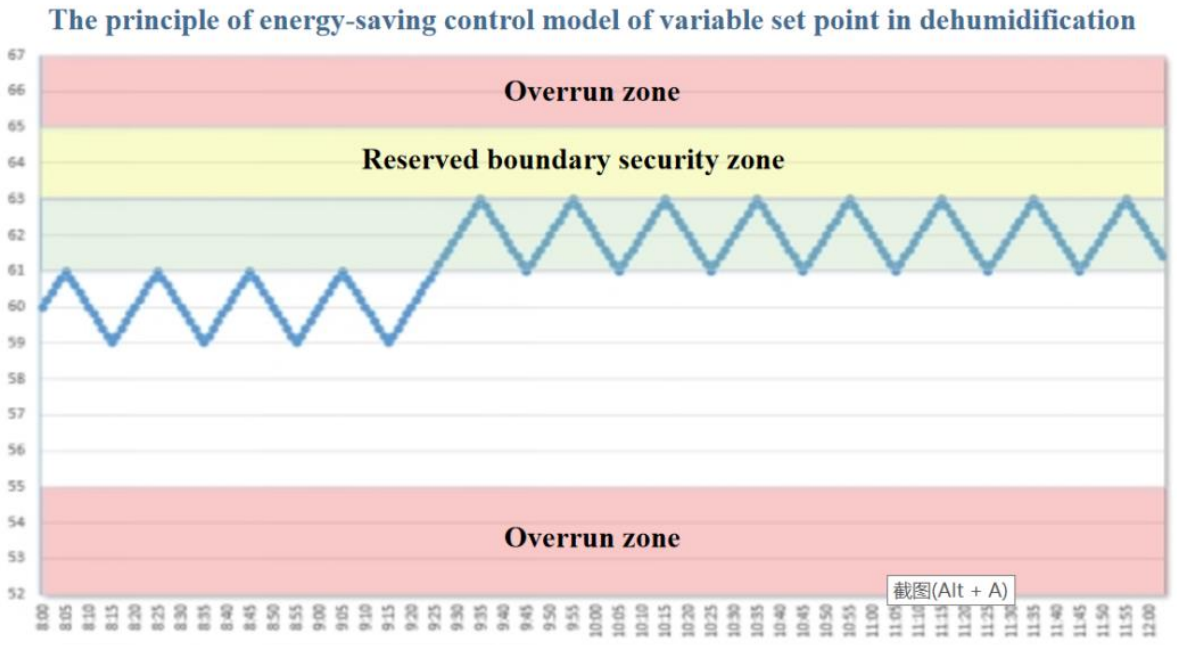

Figure 9:Energy-saving control model of variable set point in dehumidification conditions

In the refrigeration and dehumidification mode, the central value of the humidity control target of the air conditioning control system is $60 \%$, it is assumed that the maximum fluctuation range of the system humidity in the past 2 hours is $\pm 1 \%$. Using an innovative energy-saving control model that automatically changes the set value, the automatic control system will automatically gradually increase the humidity set value to $62 \%$ after analyzing the trend change in the past 2 hours, then began to continuously check and analyze the fluctuation trend of environmental humidity, assuming that the maximum humidity fluctuation observed after a period of time is still $\pm 1 \%$, then the adjusted new setting value closer to the upper limit of the humidity setting value will be maintained;On the contrary, if the humidity is over-adjusted, the humidity setting value will automatically return to the center setting value step by step, and finally ensure that the reserved safety boundary value $>=$ the maximum wave range. During the entire control process, the humidity setting value of the control system is dynamically and slowly gradually changed according to the stability of the environmental humidity, in the end, the system humidity setting value can be adaptively increased as much as possible while ensuring sufficient boundary safety will not exceed the standard, so as to reduce the demand for dehumidification and cooling capacity to achieve the purpose of energy saving in air conditioning.

\section{CONCLUSION}

In the industrial field, the primary indicator of the air conditioning control system is to continuously and uninterruptedly meet the high-precision constant temperature and humidity 
requirements in the production process, and quickly and effectively overcome various external interferences. Therefore, this article adopts a variable-parameter adaptive PID control strategy. The control strategy adjusts the PID control parameters of the system under different working conditions, so that the air-conditioning system can respond quickly and ensure high control accuracy. In addition, this article adopts a dynamic set value adaptive energy-saving control strategy, the core of the control strategy is to make reasonable use of the allowable deviation zone of the process environmental indicators, and achieve better air-conditioning energy-saving effects by controlling as close as possible to the lower limit in winter and the upper limit in summer without exceeding the requirements of the maximum process index. Through the comprehensive energy-saving control strategy of the air-conditioning system adopted in this paper, the energy-saving requirements of the air-conditioning system can be achieved under the premise of ensuring the control accuracy of the air-conditioning system.

\section{REFERENCES}

[1] Sun YiJian(2000)Research on Air Conditioning in Cigarette Factory.HAVC:12-14

[2] Huang HangChang(2005)Research and Application of Energy-saving Control Strategy for Air-conditioning System in Cigarette Factory.Energy and environment:29-31

[3] Ma JingGuo(1992)New Energy Technology.Nanjing:Jiangsu Science and Technology Press.

[4] Liang ChunSheng(2008)Energy-saving technology of central air-conditioning meter flow control.Beijing:Publishing House of Electronics Industry.

[5] Tan Ji(2018)Analysis on the control strategy of energy-saving design of central air-conditioning in large public buildings.Intelligent Building And Smart City:44-45

[6] Rao GuangYang, Cao WeiZhong(2017)Energy-saving design of central air-conditioning water circulation system.Engineering Technology Research:4-6

[7] Cai ShaoMing(2014)The energy-saving design of central air-conditioning system.Technological Innovation and Application:232-233

[8] She YiJun(2013)Energy-saving design of constant temperature central air-conditioning system.Electronic world:136-137

[9] Wang YanWei, Ji ZhiFei,Li GuangDi(2009)Energy-saving design of central air-conditioning system.Practice and Understanding of Mathematics:213-218 